\title{
Silent exonic mutation in the acid- $\alpha$-glycosidase gene that causes glycogen storage disease type II by affecting mRNA splicing
}

\author{
Mireguli Maimaiti, Satoru Takahashi, Kazuki Okajima, Nao Suzuki, Junko Ohinata, Akiko Araki, \\ Hajime Tanaka, Tokuo Mukai and Kenji Fujieda
}

Glycogen-storage disease type II (GSDII) is an autosomal recessive disorder caused by a deficiency of acid $\alpha$-glucosidase (GAA). The residual GAA activity is largely related to the severity of the clinical course. Most patients with infantile-onset GSDII do not show any enzyme activity, whereas patients with the late-onset forms of GSDII show various degrees of GAA activity. We performed a molecular genetic study on a Japanese boy with childhood-onset GSDII. The patient was a compound heterozygote for a newly discovered splice-site c.546G $>$ T mutation and a recurrent missense p.R600C mutation, which usually causes the fatal infantile form in a homozygous state. The c.546G $>$ T mutation, which did not alter the amino-acid sequence, was positioned at the last base of exon 2. cDNA-sequencing analysis revealed that c.546G $>T$ was a leaky splice mutation, leading to the production of a normally spliced transcript, which was responsible for the low-level (approximately 10\%) expression of the active enzyme in the patient's fibroblasts.

Journal of Human Genetics (2009) 54, 493-496; doi:10.1038/jhg.2009.66; published online 17 July 2009

Keywords: acid $\alpha$-glucosidase; glycogen-storage disease type II; mutation; Pompe disease; splicing

\section{INTRODUCTION}

Pompe disease (OMIN no. 232300), also known as glycogen-storage disease type II (GSD II), is characterized by autosomal recessive inheritance and lysosomal glycogen accumulation caused by a deficiency in the acid $\alpha$-glucosidase enzyme (GAA). ${ }^{1}$ GSDII shows various phenotypic variants, ranging from a rapidly fatal infantile-onset form to a slowly progressive late-onset form. The infantile form, which is manifested in the first few months of life, is characterized by severe hypotonia, progressive weakness and massive cardiomegaly, and causes cardiac and respiratory failure before 2 years of age. In contrast, the late-onset forms usually manifest as a slowly progressive myopathy associated with respiratory insufficiency, which is generally limited to skeletal muscles and is without cardiac symptoms. ${ }^{1}$

The GAA-encoding gene is located on chromosome 17q25.2-q25.3 and contains 20 exons, the first of which is noncoding. ${ }^{2}$ The GAA cDNA contains a 2856-bp-long coding sequence and encodes a 952-amino-acid polypeptide with a calculated molecular mass of $105 \mathrm{kDa}^{3,4}$ The enzyme is synthesized as a catalytically inactive precursor and undergoes posttranslational modification. ${ }^{5,6}$ The precursor protein then undergoes a stepwise proteolysis at both termini, yielding the mature GAA consisting of four associated polypeptides. ${ }^{4}$

We conducted a molecular genetic study of a Japanese patient with childhood-onset GSDII. The patient was found to be a compound heterozygote for a recurrent missense mutation and for a newly discovered splice-site mutation of the GAA gene.

\section{MATERIALS AND METHODS}

Case report

The patient, a boy who is now aged 9 years, was the first child of healthy unrelated Japanese parents. The patient was born at term after an uneventful pregnancy. The patient was healthy in the neonatal period. His early motor development was not delayed: he could walk unaided at the age of 1 year 3 months. At the age of 3 years, the patient suffered from influenza, and he was found to show elevated creatine kinase levels $\left(1500-3000 \mathrm{IU}^{-1}\right)$. An examination revealed mild muscle weakness with proximal dominance. As there were no deletions in the dystrophin gene, the patient underwent muscle biopsy at the age of 5 years. The biopsied skeletal muscle showed a marked accumulation of vacuolar glycogen. An enzyme assay revealed a reduced activity of GAA in the skin fibroblasts of the patient $\left(0.76 \mathrm{nmol} \mathrm{mg}^{-1}\right.$ protein per $30 \mathrm{~min}$, which was $10.4 \%$ of the average control activity $\left(7.3 \pm 2.2 \mathrm{nmol} \mathrm{mg}^{-1}\right.$ protein per $30 \mathrm{~min})$ ). Echocardiography did not indicate any abnormal findings. He was too young to have a formal testing of lung function performed, but he has never shown any symptoms of respiratory insufficiency. The patient is currently being treated with enzyme replacement therapy, which was initiated at 7 years of age when he exhibited mild hypotonia and difficulty in running. After 2 years of treatment, the patient exhibited a good clinical response with a partial recovery of exercise abilities.

\section{Mutational analysis of the $G A A$ gene}

After obtaining informed consent, we extracted genomic DNA from the peripheral blood leukocytes of the patient and his mother, and we used the DNA samples as templates for PCR. We could not obtain DNA samples from his father. DNA fragments spanning the entire GAA-coding region and the 
Table 1 Oligonucleotide primers for PCR and sequencing

\begin{tabular}{|c|c|c|c|c|}
\hline No. & Target & Nucleotide & $n$ & Orientation \\
\hline 1 & Exon 2 & 5'-CGCGGTTGATGTCTCAGAGCTGC-3' & Intron 1 & Sense \\
\hline 2 & Exon 2 & -ACCCCACCCTTGTGAGGTGC-3' & & \\
\hline 3 & Exon 2 & & & \\
\hline 4 & Exon 2 & -GCTTTGCAGGGATGTAGCA-3' & & ntisense \\
\hline 5 & Exon 2 & - ACCTTCTTCCCCAAGGACAT-3' & xon 2 & Sense \\
\hline 6 & Exon 2 & CCATTGTCTGCTCACACCTC-3' & & Antisense \\
\hline 7 & Exon 3 & 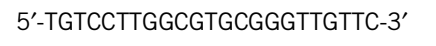 & 2 & \\
\hline 8 & Exon 3 & ССТССССАТСАТGСТ & tron 3 & ntisense \\
\hline 9 & $\begin{array}{l}\text { Exons } 4 \\
\text { and } 5\end{array}$ & '-GGTGCTCTCTGGGTGCTCTCAGG-3' & tron 3 & Sense \\
\hline 10 & $\begin{array}{l}\text { Exons } 4 \\
\text { and } 5\end{array}$ & $3^{\prime}$ & & Antisense \\
\hline 11 & Exon 6 & $3^{\prime}$ & $\ln$ & e \\
\hline 12 & & & & \\
\hline 13 & $\begin{array}{l}\text { Exons } 7 \\
\text { and } 8\end{array}$ & & & \\
\hline 14 & & $3^{\prime}$ & 8 & A \\
\hline 15 & Exon 9 & & & \\
\hline 16 & Exon 9 & $3^{\prime}$ & & ense \\
\hline 17 & & 5 & & 0 \\
\hline 18 & $\begin{array}{l}\text { Exons } 10 \\
\text { and } 11\end{array}$ & 5'-GTGCTAAGTCTCCCAGGCCAGA-3' & Intron 11 & Antisense \\
\hline 19 & Exon 12 & 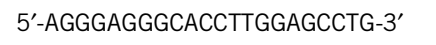 & 11 & e \\
\hline 20 & Exon 12 & & & \\
\hline 21 & Exon 13 & 5 & 12 & Sense \\
\hline 22 & Exon 13 & $5^{\prime}$ & 13 & Antisense \\
\hline 23 & Exon 14 & & & 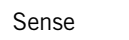 \\
\hline 24 & Exon 14 & & 14 & Antisense \\
\hline 25 & Exon 15 & 5'-TGAGA & 14 & Sense \\
\hline 26 & Exon 15 & 5'-GACAGGGCTGCCTGGGAGTTACG-3' & Intron 15 & Antisense \\
\hline 27 & Exon 16 & 5'-ATTCAGCCT & Intron 15 & Evose \\
\hline 28 & Exon 16 & 5'-TTTCCGCCACCTGGTCACCAAC-3' & Intron 16 & Antisense \\
\hline 29 & Exon 17 & 5'-GGGAGATGGAGAGCGTGGTTCC-3' & Intron 16 & Sense \\
\hline 30 & Exon 17 & 5'-СTСССССАССАТСТСССТGTGC-3' & Intron 17 & Antisense \\
\hline 31 & Exon 1 & 5'-TGCTGTACCAGCCTAGCATTCCC-3' & Intron 17 & Sense \\
\hline 32 & Exon 18 & & Intron 18 & Antisen \\
\hline 33 & Exon 19 & 5'-CCAGCTGTCTGCTGACACCT-3' & Intron 18 & Sense \\
\hline 34 & Exon 1 & АССТTCTGC-3' & Intron 19 & Antisense \\
\hline 35 & & & & (IIT) \\
\hline 36 & Exon 20 & 5'-TGGATACATCCTCCCTGCCCTG-3' & Intron 20 & Antisen \\
\hline
\end{tabular}

intron-exon boundaries were obtained using appropriate primers, as listed in Table 1. PCR fragments were analyzed using automated sequencing (GenBank accession number of reference sequence, Y00839.1).

\section{RNA analysis}

Total RNA was extracted from peripheral blood cells using the PAXgene Blood RNA Kit (Qiagen GmbH, Hilden, Germany). cDNA was generated using $1 \mu \mathrm{g}$ of total RNA in a $20-\mu$ l reaction mixture (SuperScript First-Strand Synthesis System, Invitrogen, Carlsbad, CA, USA). The sequences encompassing the three DNA variants found in this patient were amplified by PCR with $1 \mu$ of the cDNA preparation and the following primers: for c.199G $>A$ in exon 2, forward, 5'-CATCTCCAACCATGGGAGTG-3' and reverse, 5'-CACTGTTCC TGGGTGATGGC- $3^{\prime}$; for c.546G $>$ T in exon 2, forward, $5^{\prime}$-CATCTCCAACCA TGGGAGTG-3' and reverse, 5'-GAACTCCACGCTGTAGAGTG-3'; for c.1798C $>\mathrm{T}$ in exon 13, forward, 5'-CAATGAGCTGGAGAACCCAC-3' and reverse, 5'-CAGCTCCTCTGAGGTGTTGC-3'. The PCR products were electrophoresed on a 1.5\% agarose gel, visualized by ethidium bromide staining and then analyzed using automated sequencing.

\section{RESULTS}

Identification of the recurrent missense mutation in the GAA gene of the patient

Direct sequencing revealed three heterozygous DNA variants in this patient: two missense mutations and a silent exonic mutation (Figure 1). These sequence variants were not detected in 52 control genomic DNA samples. The patient's genome carried two missense mutations: c.199G $>$ A in exon 2, a predicted substitution of aspartic acid by asparagine (p.D67N), and c.1798C $>\mathrm{T}$ in exon 13, a predicted substitution of arginine by cysteine (p.R600C). These two missense mutations were maternally derived. The silent exonic variant, c.546G $>\mathrm{T}$, which does not alter the amino-acid sequence, may be paternally derived. The first missense mutation, c.199G $>$ A (p.D67N), was found at amino acid position 67 , where the aspartic acid residue is not conserved among different species (data not shown). Moreover, a small polypeptide (amino acids 57-78) has been shown to undergo cleavage during GAA maturation. ${ }^{4}$ However, several other pathogenic mutations in the GAA gene have been reported to be located in the regions that are proteolytically removed during the maturation of the enzyme. ${ }^{7}$ Therefore, further functional studies may be required to clarify the association between this mutation and GSDII. The second missense mutation, c.1798C $>$ T (p.R600C), has been identified in 8 of 22 Japanese patients. ${ }^{8}$

\section{Identification of a novel donor splice-site mutation in the GAA gene of the patient}

The c.546G $>$ T mutation, which does not result in an amino-acid change (threonine at amino acid position 182), is positioned at the last base of exon 2. However, this silent exonic variant seemed to modify the donor splice site (ACGgtgggc was modified to ACTgtgggc), because the assessment using the splice-site prediction program (http://www.fruitfly.org/seq_tools/splice.html) revealed that the probability score for this exonic variant was lower than that for the naturally occurring splice signal. In the genomic DNA sequencing, the patient was found to be heterozygous for the c.546G $>$ T mutation (Figure 1b). However, in the cDNA sequencing, he presented as a homozygote for c.546G, with the low-level expression of the c.546T allelic transcript (Figure 2b). This finding indicated that the c.546G $>$ T mutation is a splice-site mutation, and this mutation hinders the detection of a stable messenger. We did not detect any abnormal splice products. Moreover, cDNA sequencing analysis at nucleotide positions c.199 and c.1798 revealed that the signal intensities of the paternal-allele-derived transcript with the c.546G $>\mathrm{T}$ mutation were weaker than those of the maternal-allele-derived transcript (Figure 2a and c). These findings confirmed that c.546G $>\mathrm{T}$ is a leaky splice mutation by which a normally spliced transcript is produced from the mutant allele.

\section{DISCUSSION}

In this study, we report that compound heterozygous sequence variants (c. 546G $>\mathrm{T}$ (a leaky donor splice mutation) and c.1798C $>\mathrm{T}$ (p. R600C)) occurring on different alleles of the GAA gene caused a childhood-onset form of GSDII in a patient. A missense mutation p.R60C identified in this patient has been reported in other Japanese patients with GSDII; homozygosity for this mutation causes the infantile phenotype. ${ }^{8}$ Consistent with this clinical observation, an in vitro expression study has shown that p.R600C expressed negligible enzyme activity. ${ }^{8}$ Generally, there is a good correlation between the 

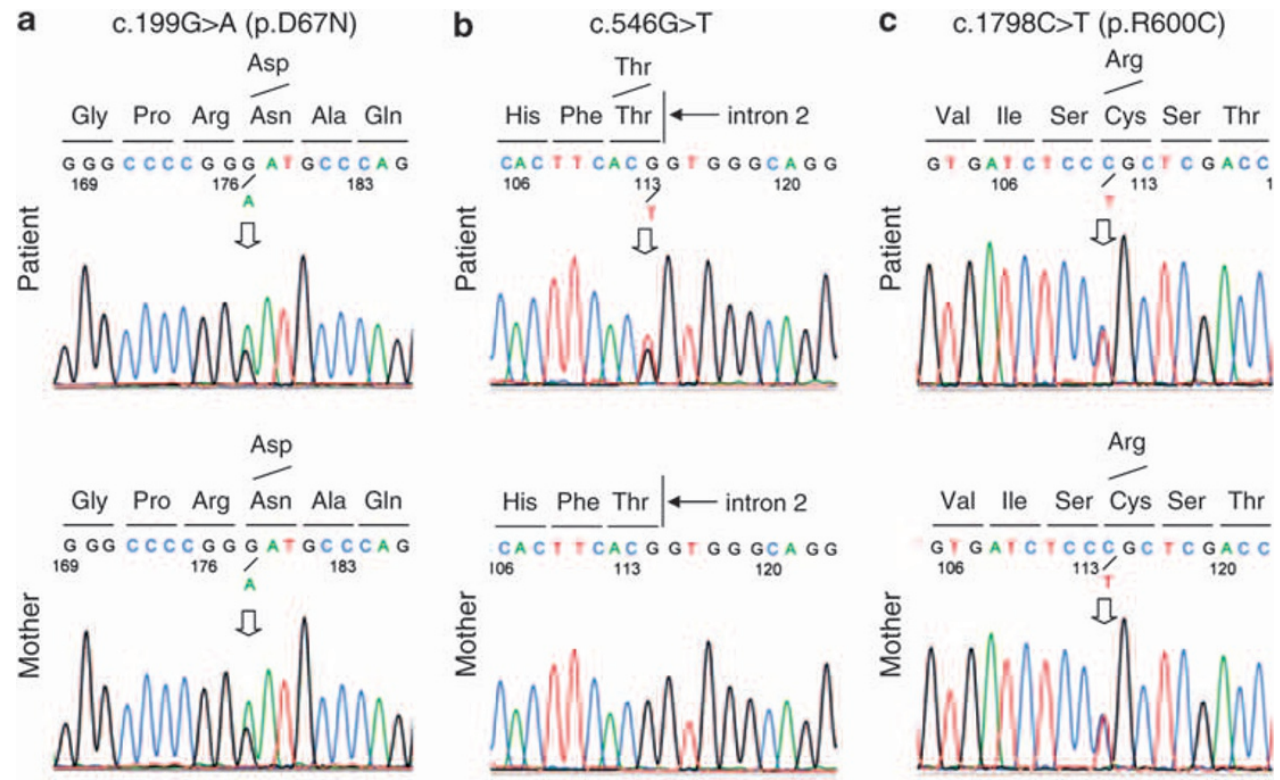

Figure 1 The three heterozygous DNA variants of the GAA gene in this patient. Automated DNA sequencing using the PCR products from this patient revealed two missense mutations and a silent exonic mutation as indicated by the arrows: C.199G $>$ A (GenBank accession number of reference sequence, Y00839.1) in exon 2, which results in an aspartic acid-to-asparagine substitution at amino acid position 67 (p.D67N) (a); c.546G > T in exon 2, which does not alter the amino-acid sequence (b); and $\mathrm{c} .1798 \mathrm{C}>\mathrm{T}$ in exon 13 , which results in an arginine-to-cysteine substitution at amino acid position 600 (p.R600C) (c). Two missense mutations, c.199G >A and c.1798C > T, of these DNA variants were also present in a heterozygous state in his mother.

a Nucleotide position c.199
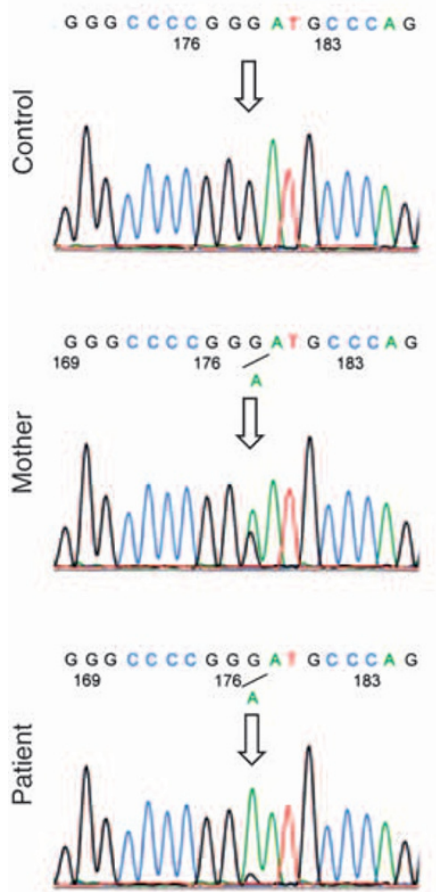

b
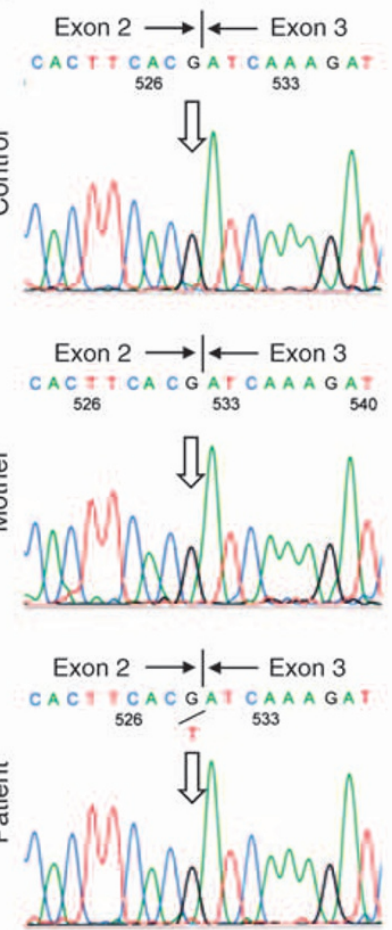

C Nucleotide position c.1798
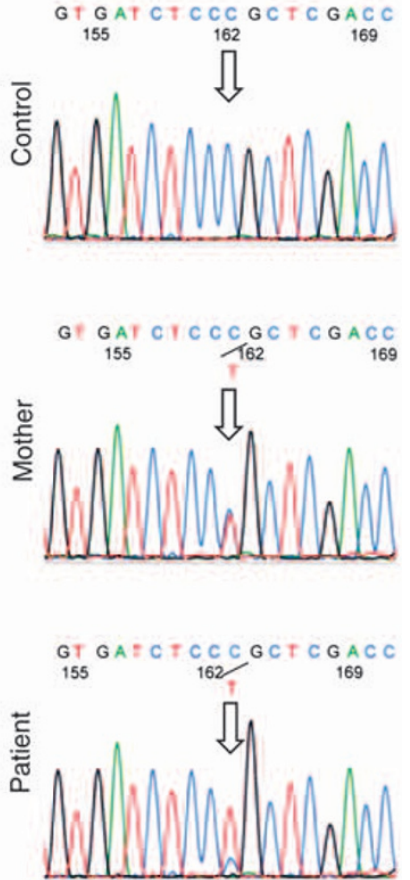

Figure 2 Silent exonic mutation c.546G $>$ T at the last base of exon 2 is a leaky splice mutation. Sequencing analysis of cDNA synthesized from total RNA demonstrates that the expression of the c.546T allelic transcript was lower than that of the c.546G allelic transcript, indicating the unstable nature of the aberrantly spliced transcript from the mutant c.546T allele (b). The 'leakiness' of this splice mutation, due to which the normally spliced transcript from the mutant allele is present at a low level, is confirmed by sequencing analysis of cDNA at nucleotide positions c.199 and c.1798 (a and c).

nature of the mutation, the degree of residual enzyme activity and the severity of clinical presentation. ${ }^{9}$ Infantile-form patients show either complete or near-complete enzyme deficiency, whereas late-onset patients retain some residual enzyme activity. This patient showed the late-onset form of GSDII. The GAA activity in the fibroblasts of this patient reduced to $10.4 \%$ of that in controls. Thus, it can be speculated 
that another mutation will compensate for the severity of the p.R600C-associated phenotype.

Another mutation, c.546G $>\mathrm{T}$, which did not result in an aminoacid change, was identified at the last base of exon 2. To date, over 200 disease-associated variants of the GAA gene have been identified in GSDII patients. This c.546G $>\mathrm{T}$ variation has never been described in the GSDII mutation database (http://www.pompecenter.nl/). However, two other base changes ( $\mathrm{G}$ to $\mathrm{C}$ and $\mathrm{G}$ to $\mathrm{A}$ ) at the same position have been described previously. ${ }^{10,11}$ Both of the c.546G $>\mathrm{C}$ and c.546G $>$ A mutations did not lead to an amino-acid substitution, but seemed to modify the splice donor site of exon 2. A Spanish patient with the c.546G $>$ C mutation presented with the adult form of this disease and had $21 \%$ residual enzyme activity in the fibroblasts. ${ }^{10}$ The patient was found to have the c.-32-13T $>\mathrm{G}$ mutation as the second genetic defect, which has been reported to be the most common among Caucasian patients with late-onset GSDII. ${ }^{12}$ The c.546G > A mutation was found in a British patient, in combination with the fully delirious c.525del mutation on the second allele. ${ }^{11}$ The patient was diagnosed at the age of 68 years and had only $3 \%$ residual enzyme activity in the fibroblasts. The c.546G $>\mathrm{T}$ variation identified in this patient also seemed to diminish the potential of the splice donor site, as the splice-site prediction program indicated. Genomic DNA sequencing showed that the $c .546 \mathrm{G}>\mathrm{T}$ variation is present in a heterozygous state in this patient. However, a sequencing analysis of the cDNA synthesized from total RNA revealed that the expression of the c.546T allelic transcript was lower than that of the c.546G allelic transcript. These findings indicated that c.546G $>\mathrm{T}$ is a leaky splice mutation. This 'leakiness' of the mutation resulted in the low-level expression of a normal messenger from the c.546G $>$ T allele, which may have been responsible for the $10 \%$ residual GAA activity in this patient's fibroblasts. This Japanese patient developed the disease much earlier in life compared with the British patient with the $3 \%$ residual enzyme activity in the fibroblasts. Thus, the levels of residual enzyme activity in the fibroblasts did not account for the different disease phenotype. It has been reported that the age of onset and disease course were different even in patients with an identical genotype. ${ }^{13}$ It is therefore conceivable that other factors such as genetic background or modifying genes may influence the clinical phenotype associated with the GAA mutation.

We report a novel splice donor mutation of the GAA gene, c.546G $>\mathrm{T}$, which, in combination with a recurrent missense mutation (p.R600C), results in the childhood-onset form of GSDII. Our findings demonstrate the importance of the last base of exon 2 (c.546G) in the determination of the splice site and the extent of missplicing caused by the mutation.

\section{ACKNOWLEDGEMENTS}

We sincerely thank the members of the family for their participation, Dr Ikuya Nonaka and Dr Ichizo Nishino for their pathological examination of the muscle and Dr Hideo Sugie for his enzyme assay. This work was supported by a grant from Takeda Science Foundation for MM.

1 Hirschhorm, R. \& Reuser, A. J. J. Glycogen storage disease type II. Acid alphaglucosidase (acid maltase) deficiency In: Scriver CR, Beaudet AL, Sly WS, Valle D (eds) The metabolic and molecular bases of inherited disease, MacGraw-Hill: New York, pp 3389-3420 (2001).

2 Kuo, W. L., Hirschhorn, R., Huie, M. L. \& Hirschhorn, K. Localization and ordering of acid alpha-glucosidase (GAA) and thymidine kinase (TK1) by fluorescence in situ hybridization. Hum. Genet. 97, 404-406 (1996).

3 Hoefsloot, L. H., Hoogeveen-Westerveld, M., Reuser, A. J. \& Oostra, B. A. Characterization of the human Iysosomal alpha-glucosidase gene. Biochem. J. 272, 493-497 (1990).

4 Moreland, R. J., Jin, X., Zhang, X. K., Decker, R. W., Albee, K. L. \& Lee, K. L. Lysosomal acid alpha-glucosidase consists of four different peptides processed from a single chain precursor. J. Biol. Chem. 280, 6780-6791 (2005).

5 Hermans, M. M., Wisselaar, H. A., Kroos, M. A., Oostra, B. A. \& Reuser, A. J. Human lysosomal alpha-glucosidase: functional characterization of the glycosylation sites. Biochem. J. 289, 681-686 (1993).

6 Wisselaar, H. A., Kroos, M. A., Hermans, M. M., van Beeumen, J. \& Reuser, A. J. Structural and functional changes of lysosomal acid alpha-glucosidase during intracellular transport and maturation. J. Biol. Chem. 268, 2223-2231 (1993).

7 Kroos, M., Pomponio, R. J., van Vliet, L., Palmer, R. E., Phipps, M., Van der Helm, R. et al. Update of the Pompe disease mutation database with 107 sequence variants and a format for severity rating. Hum. Mutat. 29, E13-E26 (2008).

8 Tsujino, S., Huie, M., Kanazawa, N., Sugie, H., Goto, Y., Kawai, M. et al. Frequent mutations in Japanese patients with acid maltase deficiency. Neuromuscul. Disord. 10, 599-603 (2000).

9 Schoser, B., Hill, V. \& Raben, N. Therapeutic approaches in glycogen storage disease type II/Pompe disease. Neurotherapeutics 5, 569-578 (2008).

10 Gort, L., Coll, M. J. \& Chabás, A. Glycogen storage disease type II in Spanish patients: high frequency of c.1076-1G >C mutation. Mol. Genet. Metab. 92, 183-187 (2007).

11 Hermans, M. M., van Leenen, D., Kroos, M. A., Beesley, C. E., Van Der Ploeg, A. T., Sakuraba, H. et al. Twenty-two novel mutations in the lysosomal alpha-glucosidase gene (GAA) underscore the genotype-phenotype correlation in glycogen storage disease type II. Hum. Mutat. 23, 47-56 (2004).

12 Huie, M. L., Chen, A. S., Tsujino, S., Shanske, S., DiMauro, S., Engel, A. G. et al. Aberrant splicing in adult onset glycogen storage disease type II (GSDII): molecular identification of an IVS1 $(-13 \mathrm{~T} \rightarrow \mathrm{G})$ mutation in a majority of patients and a novel IVS10 (+1GT $\rightarrow$ CT) mutation. Hum. Mol. Genet. 3, 2231-2236 (1994).

13 Nascimbeni, A. C., Fanin, M., Tasca, E. \& Angelini, C. Molecular pathology and enzyme processing in various phenotypes of acid maltase deficiency. Neurology 70, 617-626 (2008). 